Genre et filiation : pratiques et représentations

\title{
Tu quoque, mi fili !
}

Pourquoi les Français croient-ils que Brutus était le fils de César?

Why do the French believe that Brutus was Caesar's son?

\section{Marine Bretin-Chabrol}

\section{(2) OpenEdition}

Journals

\section{Édition électronique}

URL : http://journals.openedition.org/transtexts/492

DOI : $10.4000 /$ transtexts.492

ISSN : 2105-2549

Éditeur

Gregory B. Lee

Référence électronique

Marine Bretin-Chabrol, «Tu quoque, mi fili ! », Transtext(e)s Transcultures 跨文本跨文化 [En ligne], 8 |

2013, mis en ligne le 02 décembre 2013, consulté le 10 décembre 2020. URL : http://

journals.openedition.org/transtexts/492 ; DOI : https://doi.org/10.4000/transtexts.492

(c) Tous droits réservés 


\section{Transtext(e)s Transcultures 跨文本跨文 化}

Journal of Global Cultural Studies

8 | 2013 :

Genre et filiation : pratiques et représentations

Langage et parenté, langue maternelle, question du nom

\section{Tu quoque, mi fili ! \\ Pourquoi les Français croient-ils que Brutus était le fils de César?}

Why do the French believe that Brutus was Caesar's son?

Marine Bretin-Chabrol

\section{Résumés}

Français English

Marcus Junius Brutus, qui participa au meurtre de César lors des Ides de Mars 44 av. J.-C., n'était pas le fils adoptif de ce dernier, contrairement à ce que véhicule une tradition scolaire bien établie en France. La célèbre citation latine Tu quoque, mi fili ! est elle-même truquée puisque, selon Suétone (Vie de César, 82, 3), les mots prononcés par César reconnaissant Brutus parmi les conjurés auraient été dits en grec. Déformant une rumeur rapportée par Plutarque (Vie de Brutus, 5, 2), la tradition française a en effet considéré comme le fils adoptif de César un jeune homme qui était le fils de sa maîtresse Servilia, et peut-être par conséquent son fils biologique. Dans cette version de l'histoire, l'assassinat du dictateur est dramatisé par la dimension tragique du parricide. Mais cette lecture des événements repose sur une définition anachronique du crime de parricidium (en l'occurrence, meurtre du prince et non meurtre du père) et fait contresens par rapport aux motivations que les historiens anciens prêtent à Brutus, qui tue le tyran pour se conformer à l'idéal républicain incarné par les hommes illustres de sa famille, le premier Brutus et $\mathrm{C}$. Servilius Ahala, ancêtres illustres de son père légitime et de son père adoptif, et Caton d'Utique, son oncle et beau-père. César, le tyran, est au contraire celui qui confond public et privé et porte un titre controversé, celui de père de la patrie.

Marcus Iunius Brutus, who took part in the murder of Caesar (Ides of March, 44 B.C.) was not his adopted son, although a well established school tradition may pretend he is. The well known quotation itself, tu quoque, mi fili! is a fake, since, according to Suetonius (Caes. 82, 3) Caesar's words, as he recognised Brutus among the conjurers, were in Greek. Twisting a statement 
rumoured by Plutarchus $(\mathrm{Br} .5,2)$, the French tradition has indeed made a young man who was the son of Caesar's lover Servilia, and therefore, possibly, his biological son, into Caesar's adopted son. In that version, the dictator's murder is made more dramatic because of the tragical side of the parricide. But this interpretation of the events rests on an anachronistic definition of the crime of parricidium (here meaning murder of the prince rather than of the father) and seems fatally flawed if one considers the actual motives which ancient historians lend to Brutus: he kills the tyrant in order to follow a Republican ideal which was typified by great members of his family, the first Brutus and C. Servilius Ahala, illustrious ancestors of his legitimate and his adoptive fathers, and Cato of Utica, his uncle and father-in- law. On the contrary, Caesar, the tyrant, confuses the public and the private and wrongly awarded the title of Pater patriae.

\section{Texte intégral}

En France, on apprend souvent que parmi les assassins de Jules César, le jour des Ides de mars 44 av. J.-C., se trouvait Brutus, son prétendu « fils adoptif ». Au seuil de la mort, le dictateur, frappé par cette trahison, se serait même exclamé, dit-on, Tu quoque, mi fili ! " Toi aussi, mon fils ! $»^{1}$ Ce parricide tragique offre un modèle encore vif aujourd'hui : souvenons-nous des images employées tout récemment par la presse pour évoquer la trahison du dauphin de Silvio Berlusconi, Angelino Alfano. La référence à Brutus émane peut-être de la presse italienne, mais l'idée d'une filiation adoptive entre le dictateur et son assassin, affirmée par le correspondant de l'Agence Reuters à Rome, a été reprise par plusieurs médias français :

"Alfano trahit", s’indigne jeudi en "une" Il Giornale, le quotidien du frère de Silvio Berlusconi. Pour de nombreux sympathisants du "Cavaliere", Alfano est un nouveau Brutus, le fils adoptif de César qui n’hésita pas à participer au complot des Ides de Mars. ${ }^{2}$

Or, l'authenticité historique de la citation comme du lien de parenté qu'elle suggère est remise en question depuis longtemps. Il est d'abord avéré que Brutus n'a jamais été adopté par César. D’autre part, à supposer que César ait bien adressé quelques paroles à Brutus avant de mourir, celles-ci n'auraient pas été prononcées en latin, mais en grec. C'est en effet en grec que Suétone et Dion Cassius rapportent les mots du dictateur, tout en les présentant, bien après les événements, comme une tradition possible mais non certaine. ${ }^{3}$ Tel est le récit de Suétone :

\footnotetext{
Atque ita tribus et uiginti plagis confosus est, uno modo ad primum ictum gemitu sine uoce edito, etsi tradiderunt quidam Marco Bruto irruenti dixisse « xaì ov̀

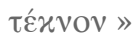

Il [= César] fut ainsi percé de vingt-trois blessures, n'ayant poussé qu'un gémissement au premier coup, sans une parole ; pourtant, d'après certains, il aurait dit à Marcus Brutus qui se précipitait sur lui : « Toi aussi, mon fils ! »4
}

La formule latine Tu quoque, mi fili! ne se rencontre pas dans les textes anciens : il s'agit d'une traduction inexacte de la formule grecque, proposée par un latiniste français du XVIIIe siècle, l'abbé Lhomond, dans le manuel qu'il constitua, en 1779, à l'usage des classes de 6e.5 Mais elle a connu une fortune immense, en partie parce qu'elle fournissait l'exemple grammatical de deux vocatifs irréguliers, $m i$ et fili, dans l'apprentissage de la deuxième déclinaison des noms et de la première classe d'adjectifs. ${ }^{6}$

En contestant l'authenticité de la formule latine bien connue et l'existence d'un lien adoptif entre César et Brutus, je ne prétends pas apporter une information nouvelle aux chercheurs. Dans le milieu universitaire, sans parler des historiens allemands, anglais ou américains qui n'ont jamais commis cette erreur, ${ }^{7}$ les anciens biographes de César et de Brutus que furent Jérôme Carcopino ou Gérard Walter ne s'y sont pas non plus trompés. ${ }^{8}$ 
Plus récemment, les articles de Michel Dubuisson et de Pascal Arnaud, respectivement parus dans la revue Latomus en 1980 et 1998, ont repris la question à nouveaux frais sans aucune ambiguïté sur la nature des liens qui pouvaient unir César et Brutus. ${ }^{9} \mathrm{M}$. Dubuisson, qui soutient la thèse d'une authenticité de la formule grecque, interroge le dossier dans le contexte de son étude du bilinguisme à Rome : prononcés sous le coup de l'émotion, les derniers mots de César l'auraient été dans la langue qui lui était la plus familière, la plus intime, le grec. P. Arnaud voit au contraire dans cette formule le pastiche d'une citation littéraire qu'une tradition hostile à Brutus aurait placé $a$ posteriori dans la bouche de César pour faire apparaître le geste de ses meurtriers comme un acte plus ambitieux que désintéressé : «Toi aussi, mon fils, tu mangeras une part de notre pouvoir ! » aurait ainsi prophétisé le dictateur mourant.

Malgré ces mises au point régulières, ${ }^{10}$ la presse n'est pas la seule à véhiculer encore cette histoire de filiation adoptive : il y a comme une résistance, presque une dénégation, du public français cultivé à entendre les faits. Voici par exemple comment Max Gallo ( « historien mais aussi, d'évidence, confident du héros », pour citer la 4e de couverture du livre) relate la fin de César dans sa biographie du grand homme :

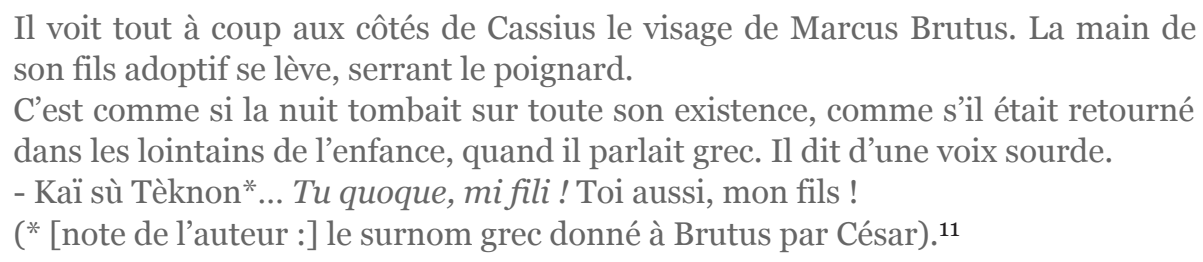

Alors que l'évocation d'une langue grecque de l'enfance laisse penser que Max Gallo a pu lire l'article de M. Dubuisson, l'auteur maintient l'hypothèse d'une filiation adoptive entre César et Brutus. ${ }^{12}$ Mieux, alors qu'il cite l'expression grecque en la translittérant, il ne peut écarter la formule latine communément reçue par la tradition, qu'il reprend du reste comme titre de son chapitre, p. 433.

La croyance en ce préjugé est si intimement enracinée chez les Français s'intéressant à l'Antiquité qu'elle vient même entacher des travaux de haut niveau scientifique, comme l'édition des Vies parallèles de Plutarque, sous la direction de F. Hartog, parue chez Quarto en 2011. Alors qu'il ressort clairement de la Vie de Brutus par Plutarque et de son apparat critique que Brutus n'a pas été adopté par César, l’index général, p. 2187, s. $v$. Brutus énonce :

Marcus Junius Brutus, Fils adoptif et meurtrier de César (85 ?-42 av. J.-C.).

Tout cela m'a intriguée. J'ai voulu comprendre les raisons de cette confusion. N'étant pas historienne de la période contemporaine, je me suis focalisée sur le dossier ancien de façon à montrer quels étaient les facteurs de contresens possibles pour un moderne dans cette affaire. Et il m'est apparu que la question pouvait intéresser des spécialistes de gender studies au sens où l'erreur repose, à mon avis, sur une mauvaise compréhension des normes sociales qui, à Rome, régissent le comportement d'un homme issu d'une lignée sénatoriale et l'attitude qu'il se doit d'avoir envers tous les hommes avec lesquels il entretient un lien de filiation ou de quasi-filiation.

\section{Quels liens unissaient-ils Brutus et César?}

En résumant Suétone à l'usage des collégiens, l'abbé Lhomond pouvait-il traduire $\tau \varepsilon ́$ kvov par filius sans trahir la formule originale? Le terme latin est un terme de parenté 
exprimant en général, à l'époque classique, la situation juridique d'un fils par rapport à son père. C'est le mot que l'on emploie, sous une forme abrégée, dans la nomenclature officielle, comme en atteste par exemple son emploi dans l'épitaphe de Gnaeus Cornelius Scipion Hispanus :

\section{Cn. Cornelius Cn. f.[= filius] Scipio Hispanus, \\ Gnaeus, fils de Gnaeus, Cornelius Scipio Hispanus 13}

Le terme filius n'est qu'exceptionnellement employé par métaphore pour désigner le petit d'un animal, jamais pour exprimer un relation d'affection qui serait simplement analogique d'un lien de filiation. ${ }^{14}$ En revanche, le grec $\tau \dot{\varepsilon} \kappa v o v$, bien qu'il puisse aussi désigner un fils légitime, se trouve également employé dans des contextes plus vagues : dans l'épopée homérique ou dans la tragédie, un personnage d'âge mûr peut ainsi apostropher un personnage plus jeune, ou un roi ses sujets, sans que la relation affectueuse suggérée par le terme ne repose sur un lien de parenté effectif. ${ }^{15}$ En traduisant $\tau \dot{\varepsilon}$ Kvov par filius, l'abbé Lhomond a donc opéré un choix très réducteur parmi les différents sens de $\tau \dot{\varepsilon}$ kvov: par cette traduction il a établi une filiation entre César et Brutus. Or si César a bien prononcé la phrase grecque en voyant Brutus parmi les conjurés, à quel type de lien pouvait-il faire allusion?

Marcus Junius Brutus est le fils légitime du patricien M. Junius Brutus et de sa femme Servilia. ${ }^{16}$ La date de sa naissance est discutée : 85 av. J.-C., date la plus généralement admise, selon le témoignage de son ami Cicéron, ${ }^{17}$ ou bien 78 av. J.-C. selon l'historien d'époque tibérienne Velleius Paterculus. ${ }^{18}$ Son père meurt en 78 , assassiné sur l'ordre de Pompée. ${ }^{19}$ L'enfant est élevé par son oncle, le demi-frère de sa mère, Marcus Porcius Caton, passé à la postérité sous le nom de Caton d'Utique, qui l'initie à la rhétorique et à la philosophie. ${ }^{20}$ En 59, il est adopté par un autre frère de sa mère Q. Servilius Caepio, dont il prend le nom : Q. Servilius Caepio Brutus. ${ }^{21}$ En épousant Claudia, fille d'Appius Claudius Pulcher, en 54, Brutus noue avec son beau-père un lien de pseudo-filiation qu'il renforce en servant comme questeur sous ses ordres en $53 .{ }^{22}$ Après son divorce avec Claudia, et son remariage en 45 avec sa cousine Porcia, la fille de son oncle Caton mort l'année précédente, Brutus transforme le lien éducatif et nourricier qui l'unissait à son oncle en un lien de quasi-filiation posthume. Brutus ne manque donc pas de pères : il a successivement un père légitime (M. Junius Brutus) et un père adoptif (Q. Servilius Caepio), ainsi que deux beaux-pères (Ap. Claudius Pulcher, M. Porcius Cato), le second ayant également joué auprès de lui le rôle de père nourricier. Aucun lien de paternité légal ou symbolique n'unit en revanche Brutus à César.

Mais César a été l'amant de la mère de Brutus, Servilia, et Plutarque rapporte une rumeur selon laquelle Brutus aurait pu être son fils naturel.

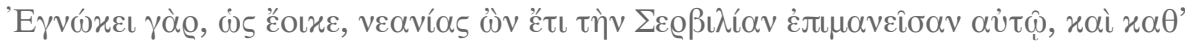

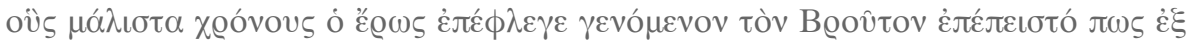

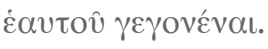

Car il paraît qu'étant encore jeune il avait eu pour maîtresse Servilia, qui était follement éprise de lui ; et, comme Brutus était né à l'époque où leur amour était le plus ardent, César avait une sorte de conviction que Brutus était de lui. ${ }^{23}$

Cependant il est difficile de connaître avec certitude le cadre chronologique des amours de César et de Servilia. On sait que César reçut de sa maîtresse un billet passionné en pleine séance du Sénat en $63^{24}$ et qu'il lui offrit une perle de grand prix lors de son premier consulat en 59.25 Etait-il déjà son amant en 85 av. J.-C., alors qu'il n'avait que 16 ans ? Bien qu'affirmée par Plutarque, cette hypothèse est généralement rejetée par les historiens modernes. ${ }^{26}$ En revanche, il est tout à fait possible que César ait eu une 
affection particulière pour un enfant qui n'était pas biologiquement le sien mais qui était le fils d'une femme qu'il aimait. Selon Plutarque, César fait en effet preuve d'une attention exceptionnelle à l'égard de Brutus quand celui-ci s'engage contre lui, aux côtés de Pompée, en 49.

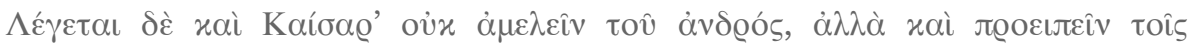

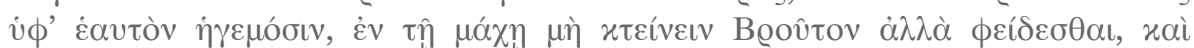

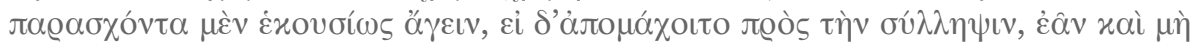

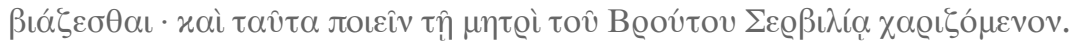

On rapporte aussi que César ne se désintéressait pas de Brutus et qu'il avait même ordonné à l'avance à ses officiers de ne pas le tuer dans la bataille, de l'épargner, et, s'il s'y prêtait de bon gré, de le lui amener, mais, s'il se défendait contre ceux qui voudraient le prendre, de le laisser aller sans lui faire violence ; il agissait ainsi, dit-on, pour complaire à la mère de Brutus, Servilia. 27
\end{abstract}

D'autres sources confirment cette bienveillance. Le futur dictateur veille à ce que son protégé ne soit pas tué. Il use d'une clémence et d'une générosité remarquables à son égard après la défaite de Pompée en $48 .^{28}$ Grâce à César, non seulement Brutus est pardonné, mais il obtient le commandement de la Gaule cisalpine fin 47 jusqu'au mois de mars $45,,^{29}$ la préture en 44,30 et une promesse de consulat pour $41^{31}$. On comprend que l'assassinat de César ait pu passer pour une trahison. S'agissait-il pour autant d'un parricide?

\title{
Un parricidium n'est pas nécessairement un parricide
}

Suétone nous apprend qu'en référence au meurtre de César, les Ides de mars furent appelées « jour parricide».

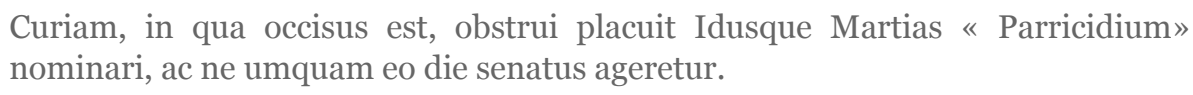

On décida de murer la curie où il avait été assassiné, de nommer les Ides de Mars « Jour Parricide », et d'interdire à tout jamais au sénat de se réunir à cette date. ${ }^{2}$

Mais il faut bien s'entendre sur le sens de ce terme. Comme l'a montré le juriste romaniste Yan Thomas, le mot a recouvert différents crimes au cours de l'histoire romaine. $33 \mathrm{Si}$, à l'origine, le terme paraît bien désigner exclusivement le meurtre d'un père, et s'il conserve généralement cette signification dans la langue courante, une loi de Pompée a étendu le crime de parricide aux meurtres perpétrés contre tous les ascendants, tous les collatéraux et tous les alliés. A partir du moment où l'on décerne le titre de "père de la patrie » au princeps, on désigne également le crime de lèse-majesté sous le nom de parricidium. Or, lorsque César est assassiné, il a reçu ce titre, qui a contribué à le rendre odieux aux défenseurs de la légalité républicaine :

\footnotetext{
Praegrauant tamen cetera facta dictaque eius, ut et abusus dominatione et iure caesus existimetur. Non enim honores modo nimios recepit : continuum consulatum, perpetuam dictaturam praefecturamque morum, insuper praenomen Imperatoris, cognomen Patris patriae, statuam inter reges, suggestum in orchestra...
}

Cependant, certains autres de ces actes et de ses discours l'emportent dans la balance, de sorte qu'il passe pour avoir abusé de la toute-puissance et mérité d'être assassiné. Il ne lui suffit pas, en effet, d'accepter des honneurs excessifs, 
comme plusieurs consulats de suite, la dictature et la préfecture des mœurs à perpétuité, sans compter le titre d'Imperator, le surnom de Père de la Patrie, une statue parmi celle des rois, une estrade dans l'orchestre...34

En tuant le " père de la patrie ", tous les conjurés, et non seulement Brutus, se sont donc rendus coupables de parricidium à l'égard du dictateur. Mais le terme de parricide a pu induire en erreur les lecteurs de Suétone aussi bien à l'époque moderne que dès l'Antiquité. Le motif du conflit familial, qu'il s'agisse de rivalités entre frères (Etéocle et Polynice, Atrée et Thyeste) ou entre père et fils (Edipe tuant Laios), est au cœur d'un imaginaire mythologique et tragique gréco-romain qui influence fortement les auteurs latins relatant, en prose ou en vers, les différents épisodes des guerres civiles de la fin de la République. 35

\title{
Un Brutus peut en cacher un autre
}

Autre facteur de confusion dans cette histoire et autre raison de douter de l'existence d'un lien de filiation ou de quasi-filiation entre Brutus et César, c'est le rôle joué par un autre Brutus, lointain cousin de celui qui nous intéresse ici, un peu plus jeune que lui. Cet homme, Decimus Junius Brutus, est le fils d'une autre femme puissante et sensuelle, Sempronia, hôtesse de quelques réunions troubles à l'époque de la conjuration de Catilina. ${ }^{36}$

Contrairement à Marcus, Decimus reste fidèle à César tout au long de sa carrière : entre 56 et 52, il s'illustre comme préfet puis légat de César lors de la guerre des Gaules, en 49 comme légat au siège de Marseille. 37 Il est gouverneur de Gaule transalpine en $48,3^{38}$ préteur en 45 , promis au consulat pour 42.39 Mais à son tour il rejoint la conjuration, lui aussi porte la main sur le père de la patrie.

\author{
Conspiratum est in eum a sexaginta amplius, Gaio Cassio Marcoque et Decimo \\ Bruto principibus conspirationis.
}

Plus de soixante citoyens conspirèrent contre lui, ayant à leur tête Gaius Cassius, Marcus et Décimus Brutus.40

L’ingratitude de ce Brutus-ci devrait être davantage encore stigmatisée par la postérité, car contrairement au personnage qui se trouve au cœur de notre réflexion, Decimus Brutus, du fait de sa fonction de légat sous les ordres de César, avait noué avec le général en chef une relation de pseudo-filiation entraînant des devoirs d'assistance mutuelle tout au long de la vie. ${ }^{41}$ Contrairement à Marcus Brutus, Decimus avait même été institué héritier par César.

sed nouissimo testamento tres instituit heredes sororum nepotes, Gaium Octauium ex dodrante, et Lucium Pinarium et Quintum Pedium ex quadrante relinquo ; in ima cera Gaium Octauium etiam in familiam nomenque adoptauit ; plerosque percussorum in tutoribus fili, si sui sibi nasceretur, nominauit, Decimum Brutum etiam in secundis heredibus.

Mais, dans son dernier testament, il institua trois héritiers, les petits-fils de ses sœurs, Gaius Octavius, pour les trois quarts, Lucius Pinarius et Quintus Pedius, pour l'autre quart ; à la fin, il déclarait même adopter Gaius Octavius, en lui léguant son nom ; il désignait plusieurs de ses assassins parmi les tuteurs du fils qui pourrait lui naître, et même Décimus Brutus parmi les héritiers de seconde ligne. 42

La postérité aurait-elle confondu les deux hommes et déduit de l'héritage de l'un la filiation de l'autre ? Ce n'est pas impossible. Quoi qu'il en soit, il semble qu'en voulant 
faire de Brutus le fils de César, la doxa scolaire a empêché de comprendre quel rôle jouait véritablement la filiation de Brutus dans son acte tyrannicide.

\section{Devenir Brutus}

Brutus était donc le fils légitime d'un homme dont la mort fut voulue par Pompée. Conformément à l'éthique aristocratique en vigueur à la fin de la République romaine, il aurait fallu que Brutus vengeât son père. 43 Malgré le soin qu'il mettait à ne pas adresser la parole à Pompée quand il le rencontrait44, malgré son implication dans une affaire d'attentat contre Pompée assez douteuse, 45 malgré une position clairement hostile au consul unique de 52,46 il ne parvint pas à se venger. Par conviction politique, il fut même contraint d'abandonner son projet de vengeance personnelle et de se rallier à la cause du meurtrier de son père parce qu'elle paraissait la moins mauvaise pour l'intérêt général, 47 et parce que c'était celle qu'avait choisie son mentor, son oncle Caton.

Mais après la défaite de Pompée à Pharsale, Brutus se laisse convaincre par César de rejoindre son camp : il laisse ainsi son oncle, Caton, à la merci des armées de César. Après la ruine de son armée et de sa cause, Caton se suicide en stoïcien. Après avoir échoué à venger son père légitime et avoir trahi sa mémoire en ralliant la cause de son meurtrier, Brutus fait ici figure de traître à son père nourricier. Cependant une nouvelle palinodie va permettre à Brutus de devenir vraiment Brutus. En épousant Porcia, la fille de Caton, il devient légitimement le vengeur de son beau-père défunt et affiche ainsi son hostilité retrouvée à l'égard de César. ${ }^{48}$

Par ailleurs, Brutus va trouver un sens à l'intérêt qu'il a toujours porté à sa généalogie : Cornélius Népos raconte en effet qu'Atticus, l'ami de Brutus et de Cicéron, aurait fait des recherches pour dresser une généalogie complète de Brutus, sans doute pour faire valoir son lien de parenté avec Lucius Junius Brutus, le fondateur de la République romaine.49 En 509 av. J.-C., à la suite du viol de Lucrèce par le fils du roi Tarquin le Superbe, Lucius Brutus, neveu du roi longtemps considéré comme faible d'esprit, a renversé la royauté.50

Du côté maternel (qui est aussi le côté de son père adoptif Q. Servilius Caepio), le meurtrier de César est également le descendant d'un autre justicier, Servilius Ahala :

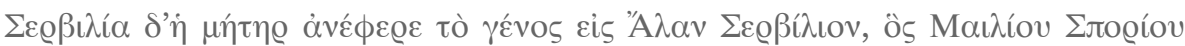

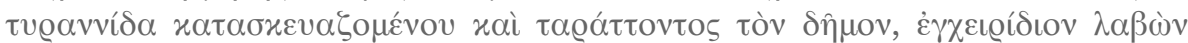

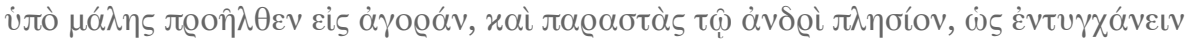

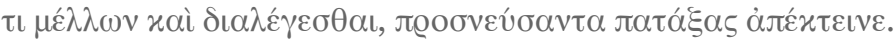

La mère de Brutus, Servilia, faisait remonter son origine à Servilius Ahala qui, voyant Spurius Moelius aspirer à la tyrannie et soulever le peuple, mit un poignard sous son aisselle, se rendit au Forum, s'approcha de lui comme pour lui parler et l'entretenir d'une affaire, et, alors qu'il penchait la tête, le frappa mortellement. $5^{1}$
}

Sous la pression de ses amis et d'écrits anonymes, Brutus va s'approprier la figure de ses deux ancêtres légendaires pour marcher dans leurs traces, maiorum exempla sequi, « suivre l'exemple des ancêtres », selon la formule usuelle. ${ }^{52}$

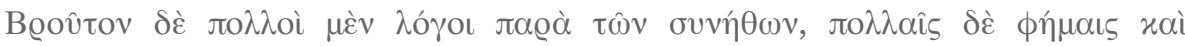

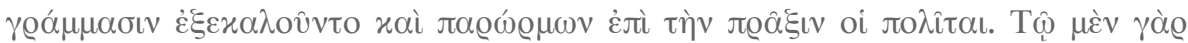

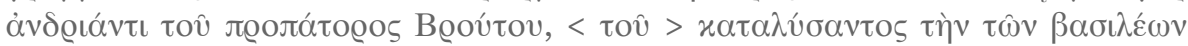

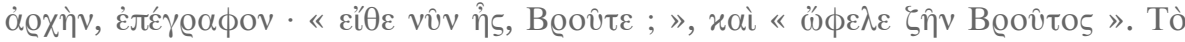

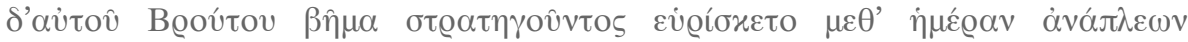

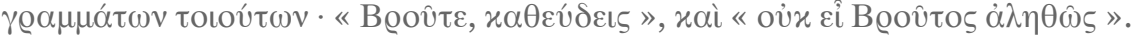

Quant à Brutus, tous les discours que lui tenaient ses familiers, et, d'autre part, 
tous les on-dit et les libelles répandus parmi les citoyens le provoquaient et le poussaient à l'action. Sur la statue de son ancêtre Brutus, qui avait aboli la royauté, on inscrivait : "Ah ! si tu existais maintenant, Brutus ! » ou «Plût au ciel que Brutus vécût ! ».53 Le tribunal de Brutus lui-même, alors préteur, se trouvait chaque jour couvert de billets tels que ceux-ci : «Dors-tu, Brutus? » ou «Tu n'es pas un vrai Brutus ».54

Aux yeux de l'aristocratie et du peuple romains, c'est le surnom de Brutus, que l'assassin de César conserve même après son adoption par son oncle maternel, qui établit symboliquement le lien de filiation malgré la distance temporelle et la méconnaissance des générations intermédiaires. Or ce lien est créateur d'obligations pour le descendant du héros d'autrefois : il doit être reconnu et, pourrait-on dire, " performé » par la réitération de l'acte héroïque accompli par l'ancêtre de la lignée.

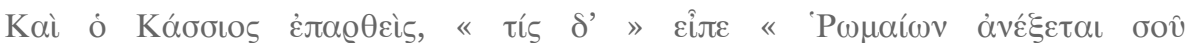

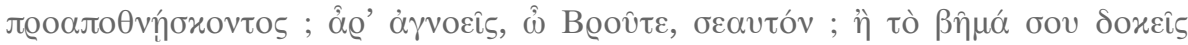

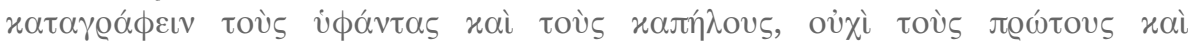

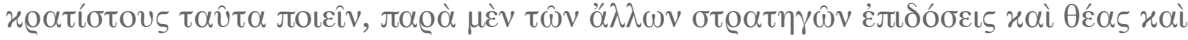

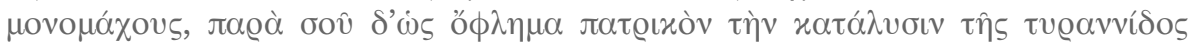

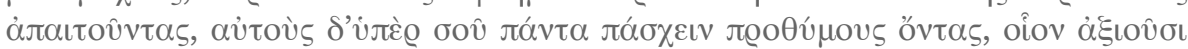

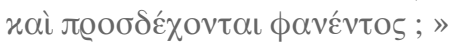

«Alors Cassius, transporté de joie, dit : « Mais quel est le Romain qui supportera de te voir mourir ainsi ? Ignores-tu, Brutus, qui tu es ? Crois-tu que ce soient les tisserands et les cabaretiers qui couvrent d'écrits ton tribunal, et non les premiers et les plus puissants des citoyens qui agissent ainsi ? Aux autres préteurs on demande des distributions, des spectacles et des combats de gladiateurs, mais ce que l'on réclame de toi, comme une dette héréditaire, c'est le renversement de la tyrannie, et les gens sont prêts à tout souffrir pour toi, si tu te montres tel qu'ils te le demandent et s'y attendent ».55

En jouant sur la célèbre formule philosophique " connais-toi toi-même ", Cassius pousse son complice à l'action. Ce n'est qu'en imitant le geste fondateur de son ancêtre, en s'acquittant d'une " dette héréditaire », que Brutus peut devenir vraiment Brutus, qu'il va faire preuve à son tour d'un courage et d'une vertu exemplaires, d'un comportement vraiment viril, c'est-à-dire digne d'un homme libre :

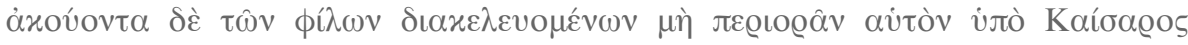

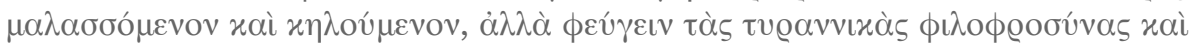

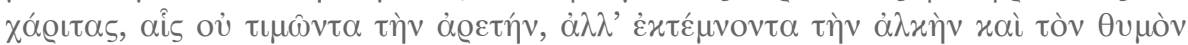

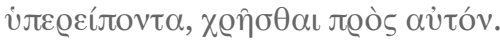

mais il écoutait les amis de celui-ci qui l'exhortaient à ne pas se laisser séduire et amollir par César et à fuir les caresses et les faveurs du tyran qui avaient pour but, non pas d'honorer sa vertu, mais d'énerver sa force et de saper son courage. 56

\title{
De pères en fils : une filiation qui se revendique dans l'action
}

Afin de tuer le tyran, Brutus a donc puisé des forces dans sa filiation : ce sont les modèles proposés par ses ancêtres, le poids de ses noms de Servilius et de Brutus, et le devoir de vengeance qu'il doit à son beau-père qui poussent Brutus à assassiner César. Face à ces figures paternelles ou ancestrales légitimes, César offre l'image d'une paternité dévoyée : non pas parce qu'il est peut-être le père naturel de Brutus - de cela, à vrai dire, tout le monde à Rome parait bien se moquer 57 - mais parce qu'il prétend gouverner le 
peuple romain comme un père exerce sa puissance sur sa famille. Le titre de " père de la patrie » est odieux aux conjurés parce qu'il rappelle que l'un des moyens par lesquels un tyran exerce sa domination est la confusion des liens publics et privés. Si les derniers mots prêtés à César donnent une couleur tragique à son assassinat, c'est peut-être aussi

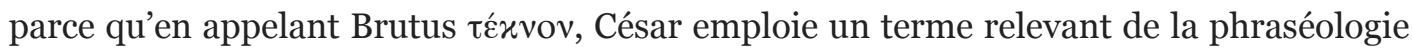
des tyrans : $\hat{\omega} \tau \dot{\varepsilon} \varkappa v \alpha$, " mes enfants », est en effet la formule qui ouvre l'EEdipe-Roi de Sophocle, et par laquelle le personnage d'Edipe s'adresse à ses sujets meurtris par la peste. Or, aux dires de Suétone, César avait lui-même composé une tragédie d'Édipe. ${ }^{8} \mathrm{Il}$ devait donc bien connaître les premiers mots de la pièce de Sophocle.

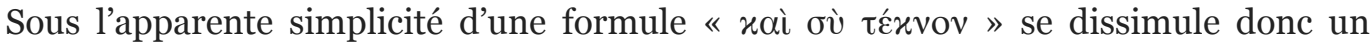
réseau complexe de relations de parenté et de pouvoir que la légende française des Ides de mars a camouflées. Je regrette de n'être pas historienne de la période contemporaine pour comprendre plus nettement pourquoi le faux-sens commis par l'abbé Lhomond a eu un tel succès dans l'imaginaire français. Certes le jeu de Goscinny avec cette formule dans quelques volumes d'Astérix a bien dû jouer son rôle, mais pourquoi la choisir ? Ce Tu quoque incorrigible a-t-il quelque chose du « lieu de mémoire »? Que dit-il de notre propre conception du pouvoir et de la filiation? du parricide tragique ? Il faudrait sans doute commencer l'enquête à la Révolution française, période pendant laquelle la vie de l'abbé Lhomond, qui avait refusé de prêter serment à la constitution civile du clergé, fut sauvée grâce à l'intervention de quelques révolutionnaires qui avaient été ses élèves, et période pendant laquelle le prénom de Brutus fut largement attribué aux nouveaux-nés.

\section{Bibliographie}

Pascal Arnaud, "Toi aussi, mon fils, tu mangeras ta part de notre pouvoir" - Brutus le Tyran ? ", Latomus 57, 1998, p. 61-71.

Catherine Baroin, "Ancestors as Models : Memory and the Construction of Gentilician Identity ", in V. Dasen et T. Späth (dir.), Children, Memory and Family Identity in Roman Culture, Oxford Clarendon Press, Oxford, 2010, p. 19-48.

Jérôme Carcopino, Jules César (1965), édition revue par Pierre Grimal, Presses Universitaires de France, Paris, 1990.

Jean-Michel David, "Maiorum exempla sequi : l'exemplum historique dans les discours judiciaires de Cicéron », MEFRM 92 (1), 1982, p. 67-86.

Michel Dubuisson, « Toi aussi, mon fils ! », Latomus 39, 1980, p. 881-89o.

Matthias Gelzer, «Iunius (Brutus) n. 53 : M. Iunius Brutus, der Caesarmörder », in PaulyWissowa, Realencyclopädie der classischen Altertumswissenschaft, X, 1, 1918, col. 978-1020.

Paul Jal, La guerre civile à Rome. Etude littéraire et morale de Cicéron à Tacite, PUF, Paris, 1963.

Philippe Moreau, «La relation de pseudo-filiation entre questeur et préteur : les vicissitudes d'un modèle politique romain tiré des relations de parenté ", in F. Thélamon (éd.), Aux sources de la puissance, sociabilité et parenté, Presses Universitaires de Rouen, 1989, p. 37-46.

Ronald Syme, « Bastards in the Roman aristocracy » (1960), in Roman Papers II, Oxford, 1979, p. 510-517.

Ronald Syme, La Révolution romaine (1960), trad. fr. de R. Stuvéras, Gallimard, Paris, 1967.

Yan Thomas, «Parricidium I. Le père, la famille et la cité », MEFRA 83 (2), 1981, p. 643-713.

Yan Thomas, « Se venger au forum. Solidarités traditionnelles et système pénal à Rome », in J.P. Poly-R. Verdier (éd.), Vengeance, pouvoirs et idéologies dans quelques civilisations de l'Antiquité, Cujas, Paris, 1984, p. 65-100.

Gérard Walter, Brutus et la fin de la République, Payot, Paris, 1939.

\section{Notes}


1 La formule a longtemps figuré dans la liste des locutions latines composant les «pages roses » du petit Larousse. Dans l'édition de 2000, p. 1097 : «Tu quoque, fili ! Toi aussi, mon fils ! Exclamation de César, lorsqu'il aperçut au nombre de ses assassins Brutus, qu'il aimait particulièrement. Cette phrase, prononcée en grec, était en réalité une imprécation ».

2 Gavin Jones (avec Paolo Biondi et Steve Scherer ; Guy Kerivel pour le service français), " Portrait - Angelino Alfano, le Brutus du "Cavaliere" », Agence Reuters, jeudi 3 octobre 2013.

3 La phrase n'est pas citée par les autres historiens rapportant l'épisode (Plutarque, Vie de Brutus, 17, 6 ; Appien, Guerres civiles, 2, 117).

4 Suétone, Vie de César, 82, 3 (sauf mention contraire, pour Suétone, la traduction choisie est celle de H. Ailloud dans la C.U.F.). Même réserve à l'égard de l'authenticité de la citation de la part de Dion Cassius, Histoire romaine, 44, 19, 5 .

5 Abbé Lhomond, De viris - Les grands hommes de Rome, trad. par Jacques Gaillard, Actes Sud, Babel, 1995, p. 358 : Quum Marcum Brutum, quem loco filii habebat, in se irruentem vidisset, dixit : «Tu quoque fili mi ! », «Quand il [= César] vit Marcus Brutus, qu'il traitait comme son fils, se précipiter sur lui, il dit : "Toi aussi, mon fils !” ».

6 Voir par exemple le manuel classique de J. Gason, A. Thomas, E. Baudiffier, Précis de grammaire des lettres latines, Editions Magnard (collection Morisset), Paris, 1979, p. 9, § 21.

7 Ronald Syme, "Bastards in the Roman aristocracy » (1960), in Roman Papers II, Oxford, 1979, p. 510-517 ; La Révolution romaine (1960), trad. fr. de R. Stuvéras, Gallimard, Paris, 1967, p. 64-65. Matthias Gelzer, "Iunius (Brutus) n. 53 : M. Iunius Brutus, der Caesarmörder », in Pauly-Wissowa, Realencyclopädie der classischen Altertumswissenschaft, X, 1, 1918, col. 9781020.

8 Jérôme Carcopino, Jules César (1965), édition revue par Pierre Grimal, Presses Universitaires de France, Paris, 1990, n. 4, p. 564 ; Gérard Walter, Brutus et la fin de la République, Payot, Paris, 1939, n. 3, p. 130-131.

9 Michel Dubuisson, « Toi aussi, mon fils ! », Latomus 39, 1980, p. 881-890 ; Pascal Arnaud, " "Toi aussi, mon fils, tu mangeras ta part de notre pouvoir" - Brutus le Tyran ? ", Latomus 57, 1998, p. 61-71.

10 Ces informations sont mises à disposition du grand public via internet, sous l'entrée « Tu quoque, mi fili »de Wikipedia (fr.wikipedia.org/wiki/Tu_quoque_mi_fili), ou dans le dossier "Quelques idées reçues à propos de Rome » proposé par le département des Sciences de l'Antiquité de l'Université de Liège (www.class.ilg.ac.be/ressources/dossiers.html).

11 Max Gallo, César Imperator, XO éditions, Paris, 2003, p. 444.

12 Max Gallo va jusqu'à inventer une scène d'adoption, op. cit. p. 122, qu'il situe dans les années 66-63 av. J.C.

13 CIL VI 1293 : l'inscription date approximativement de 130 av. J.-C.

14 Voir le Thesaurus linguae Latinae, s. v. filius.

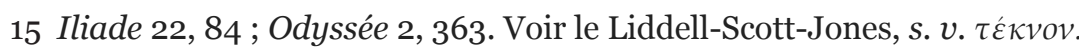

16 Plutarque, Vie de Pompée, 16, 4-8 et Vie de Brutus, 2, 1. M. Gelzer, op. cit., col. 978.

17 Cicéron, Brutus, 324. Mais le texte du passage est critiqué par certains éditeurs. Brutus est-il né « dix » (decem, selon la leçon unanime des manuscrits) ou " seize » ans (sedecim, selon la restitution de Nipperdey) avant les débuts d'Hortensius sur le forum ? La date de 85 est la plus vraisemblable si l'on admet que Brutus a été le questeur d'Appius Claudius en Cilicie en 53 av. J.-C. (Ps.-Aurelius Victor, De viris illustribus 82, 3). Cf. M. Gelzer, op. cit., col. 974 ;R. Syme, « Bastards... », p. 515.

18 Velleius Paterculus, Histoire romaine, 2, 72, 1 : Brutus serait mort à trente-six ans en 42. Une autre date se déduit encore de Tite-Live Periochae 124, pour qui Brutus serait mort à quarante ans.

19 Plutarque, Vie de Pompée, 16, 4 et 6-7.

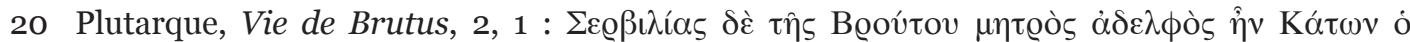

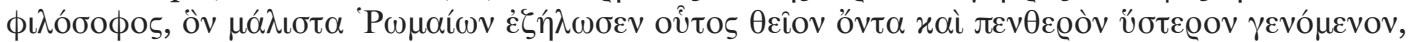
«Servilia, mère de Brutus, avait pour frère Caton le philosophe, que Brutus, son neveu et plus tard son gendre, prit comme modèle entre tous les Romains » (sauf mention contraire, pour Plutarque, la traduction choisie est celle de R. Flacelière et E. Chambry dans la C.U.F).

21 Cicéron, Lettres à Atticus, 2, 24, 2-4.

22 Cicéron, Lettres à ses familiers, 3, 4, 2 ; Brutus, 267 ; 324 ; Ps.-Aurelius Victor, De viris illustribus 82, 3. Ces liens de quasi-filiation ont été identifiés et définis par Philippe Moreau, 
«La relation de pseudo-filiation entre questeur et préteur : les vicissitudes d'un modèle politique romain tiré des relations de parenté ", in F. Thélamon (éd.), Aux sources de la puissance, sociabilité et parenté, Presses Universitaires de Rouen, 1989, p. 37-46.

23 Plutarque, Vie de Brutus, 5, 2 (trad. de la C.U.F. légèrement modifiée). Voir aussi Appien, Guerres civiles, 2, 112.

24 Plutarque, Vie de Brutus, 5, 3-4.

25 Suétone, Vie de César, 50, 4.

26 L'existence d'un lien biologique est longuement défendue par G. Walter, op. cit. ; mais contestée par J. Carcopino, op. cit., n. 4, p. 564 ; R. Syme, " Bastards... », p. 515-516 ; M. Dubuisson, op. cit., p. 884.

27 Plutarque, Vie de Brutus, 5, 1 (trad. C.U.F. légèrement modifiée).

28 Plutarque, Vie de Brutus, 6 ; Vie de César, 46, 4 ; Velleius Paterculus, Histoire romaine, 2, 52, 5 ; Dion Cassius, Histoire romaine, 41, 63, 6 ; Appien, Guerres civiles, 2, 112.

29 Plutarque, Vie de Brutus, 6 ; Cicéron, Brutus, 171 ; Lettres à ses familiers, 6, 6, 10 ;13, 10-14 ; Appien, Guerres civiles, 2, 111.

30 Cicéron, Philippiques, 10, 7 ; Lettres à ses familiers, 11, 2, 3 ; Velleius Paterculus, Histoire romaine, 2, 58,1 ; Dion Cassius, 44, 12, 3 ; Plutarque, Vie de Brutus, 7 ; Vie de César, 57, 5 ; 62, 4 ; Appien, Guerres civiles, 2, 112.

31 Plutarque, Vie de César, 62, 4 ; Cicéron, Lettres à ses familiers, 12, 2, 2 ; Philippiques, 8, 27 ; Velleius Paterculus, Histoire romaine, 2, 56, 3.

32 Suétone, Vie de César, 88, 3.

33 Yan Thomas, « Parricidium I. Le père, la famille et la cité », MEFRA 83 (2), 1981, p. 643-713.

34 Suétone, Vie de César, 76, 1-2 (trad. C.U.F. légèrement modifiée). Voir également 85, 2.

35 Paul Jal, La guerre civile à Rome. Etude littéraire et morale de Cicéron à Tacite, PUF, Paris, 1963, p. 393 ; 395-397; $401 ; 413 ; 515$.

36 Salluste, La conjuration de Catilina, 25, 1-5; 40, 5. La possibilité que Décimus Junius Brutus soit le fils naturel de César est envisagée par R. Syme, « Bastards... », p. 516-517.

37 César, La guerre des Gaules, 3, 11, 5-16, 4 ; 7, 9, 2 ; 87, 1 ; La guerre civile 1, 36, 5.

38 Appien, Guerres civiles, 2, 111.

39 Cicéron, Lettres à ses familiers, 11, 5 et 6.

40 Suétone, Vie de César, 80, 7 ; cf. aussi Plutarque, Vie de Brutus, 12, 5.

41 P. Moreau, op. cit., p. 37-46.

42 Suétone, Vie de César, 83, 3.

43 Dans ses Faits et dits mémorables, 5, 4, l'historien Valère Maxime célèbre ainsi la piété filiale (pietas) d'Aurelius Cotta, contemporain de Brutus, qui obtint la condamnation de l'homme qui avait intenté un procès à son père, consul en 74. Sur le devoir de vengeance, voir Yan Thomas, "Se venger au forum. Solidarités traditionnelles et système pénal à Rome », in J.-P. Poly-R. Verdier (éd.), Vengeance, pouvoirs et idéologies dans quelques civilisations de l'Antiquité, Cujas, Paris, 1984, p. 65-100, particulièrement p. 66-75.

44 Plutarque, Vie de Brutus, 4, 3.

45 L'affaire Vettius, en 59, à laquelle Cicéron fait référence dans une Lettre à Atticus, 2, 24, 2-4.

46 Si l'on en croit la phrase rapportée par Quintilien, Institution oratoire, 9, 3, 95 : praestat enim nemini imperare quam alicui seruire : sine illo enim uiuere honeste licet, cum hoc uiuendi nulla condicio est. "Mieux vaut n'avoir personne à qui commander que quelqu'un à qui obéir : privé de l'un, on peut vivre avec dignité, doté de l'autre, on ne peut vivre en aucune façon ».

47 Plutarque, Vie de Brutus, 4, 1.

48 Dans sa correspondance, la gêne de Cicéron à l'annonce de cet événement souligne à quel point ce mariage constitue un geste politique audacieux et dangereux, Lettres à Atticus, 13, 10, $3 ; 11,1 ; 17,1 ; 37,3 ; 48,2$.

49 Cornélius Népos, Vie d'Atticus, 18, 3. Ce lien de parenté était contesté par les adversaires de Brutus si l'on en croit Plutarque, Vie de Brutus, 1, 6-8.

50 Tite-Live, Histoire romaine, 1, 56, 7-12 (Brutus feint la stupidité) ; 59 (il déclenche la révolution).

51 Plutarque, Vie de Brutus, 1, 5-6. 
52 Jean-Michel David, «Maiorum exempla sequi : l'exemplum historique dans les discours judiciaires de Cicéron », MEFRM 92 (1), 1982, p. 67-86, particulièrement p. 82-83 ; Catherine Baroin, "Ancestors as Models : Memory and the Construction of Gentilician Identity », in V. Dasen et T. Späth (dir.), Children, Memory and Family Identity in Roman Culture, Oxford Clarendon Press, Oxford, 2010, p. 19-48.

53 Même mention chez Suétone, Vie de César, 80, 6-7.

54 Plutarque, Vie de Brutus, 9, 5-7.

55 Plutarque, Vie de Brutus, 10, 5-6.

56 Plutarque, Vie de Brutus, 7, 7.

57 Ronald Syme a montré que l'accusation de bâtardise n'était pas utilisée dans le cadre de l’invective à Rome à l'époque classique (R. Syme, « Bastards... », p. 512).

58 Suétone, Vie de César, 56, 9.

\section{Pour citer cet article}

Référence électronique

Marine Bretin-Chabrol, «Tu quoque, mi fili !», Transtext(e)s Transcultures 跨文本跨文化 [En

ligne], 8 | 2013, mis en ligne le 02 décembre 2013, consulté le 16 janvier 2014. URL :

http://transtexts.revues.org/492

\section{Auteur}

\section{Marine Bretin-Chabrol}

Marine Bretin-Chabrol est Maître de conférences de langue et littérature latine à l'Université Jean Moulin-Lyon 3 et membre du laboratoire HiSoMa (Histoire et Sources des Mondes Antiques, UMR 5189).

Marine Bretin-Chabrol is Assistant Professor in Classics at the Jean Moulin University, Lyon and a member of the research unit HiSoMa (Histoire et Sources des Mondes Antiques, UMR 5189).

\section{Droits d'auteur}

(C) Tous droits réservés 